\section{Vaccine developed to prevent periodontitis}

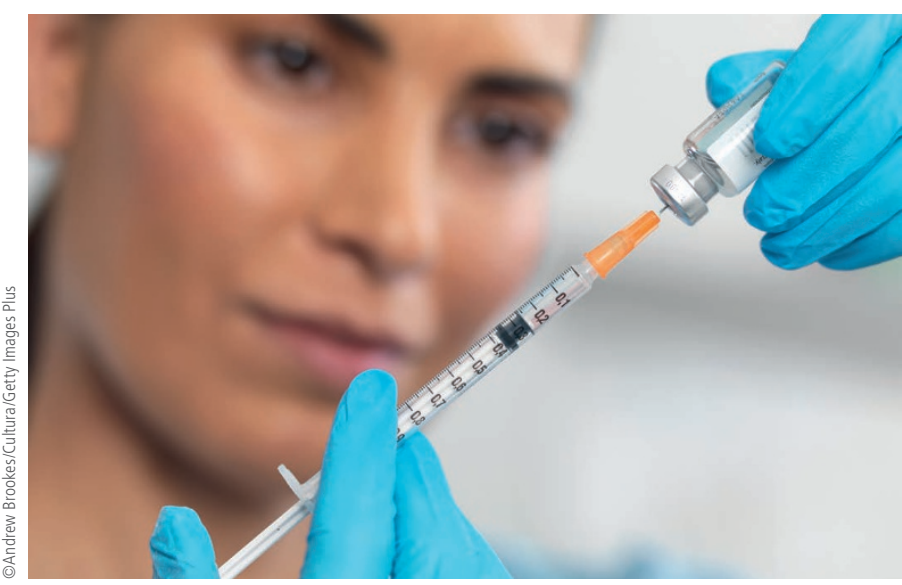

Researchers from the University of Melbourne have developed a vaccine to treat periodontitis. So far the vaccine has been tested in mice and if successful in human trials, will be able to prevent chronic periodontitis. ${ }^{1}$

The vaccine is targeted at the bacterial species that has been singled out as the main pathogen leading to gum disease. ${ }^{2}$ The vaccine will stimulate the host's immune response to produce antibodies towards this species of bacteria, preventing it from building up and reducing the inflammatory response and the level of destruction.

1. O'Brien-Simpson N M, Holden J A, Lenzo J C et al. A therapeutic Porphyromonas gingivalis gingipain vaccine induces neutralising $\lg \mathrm{G} 1$ antibodies that protect against experimental periodontitis. npj Vaccines 2016; 1: 16022; doi:10.1038/npjvaccines.2016.22; published online 1 December 2016.

2. Hajishengallis G, Darveau R P, Curtis M A. The keystone-pathogen hypothesis. Nat Rev Microbiol 2012; 10: 717-725.

\section{BOOK REVIEW}

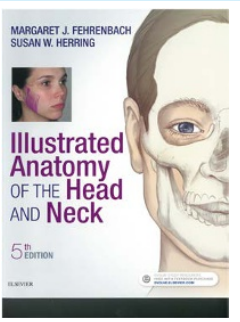

\section{ILLUSTRATED ANATOMY OF THE HEAD} AND NECK, 5TH EDITION

Margaret J. Fehrenbach and Susan W. Herring

Elsevier 2016

price $\mathrm{f} 56.69 \mathrm{pp} 352$

ISBN 9780323396349

This is the fifth edition of the Illustrated anatomy of the head and neck by authors Margaret J. Fehrenbach and Susan W. Herring.

This book has 12 comprehensive chapters and comprises over 300 pages.

The book is well-written with an appropriate level of detail which keeps the reader engaged. The start of each chapter outlines key objectives and key terms which enable the reader to direct his/her attention and also to reflect on the content of each chapter after reading. The authors aim to provide more than basic anatomy in this book and are successful in doing so. They keep the momentum throughout each chapter by using easy to read text and clever formatting, and ending each chapter with review questions and identification exercises.

\section{Oral health innovations grants available}

The Oral and Dental Research Trust (ODRT) has four new grants available to dental professionals and scientists up to a maximum of $£ 5,000$ per award.

The grants are funded by Oral Health Innovations Ltd, the UK provider of PreViser Clinical Risk and Disease Assessment technology.

The ODRT is seeking applications for small grants to support research programmes into the prevention of oral diseases with a specific emphasis on chronic diseases of ageing and patientorientated research.

Applications from the UK should comprise no more than two sides of A4 giving the background to and detail of the proposed research programme; a side of A4 detailing and justifying the financial expenditure; and an 'impact statement' to indicate how the research may benefit patients now or in the future. Applications should be submitted by email to Mrs Pam Howson, howsoncrew@aol.com,by 28 February 2017.

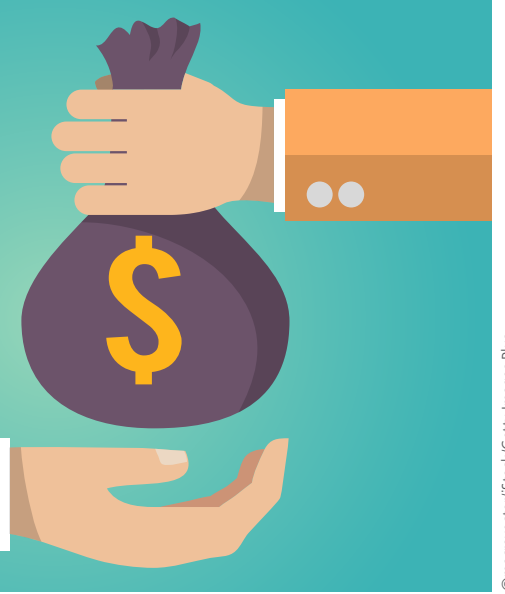

As one would expect with a book on anatomy, there are numerous images: a combination of dissections, clinical photos and colour illustrations, which work together in reinforcing a threedimensional understanding of the anatomy. The tables and flow charts used throughout the text help with the assimilation of the complex anatomy of the head and neck.

As a general dental practitioner, I particularly enjoyed reading the clinical considerations found at the end of each chapter which increased my understanding of the relevance of anatomy in clinical scenarios. The chapter on the temporomandibular joint was a delight to read as it described this complex joint and its functions in a clear, succinct manner. The authors have done a good job with the local anaesthesia and spread of infection chapters, providing clinically relevant information which would form a useful reference for any student or dental practitioner.

I would recommend this book to every medical and dental student who wishes to gain the knowledge and understanding of the anatomical structures of the head and neck, and to every dental practice as a reference text.

S. Abdul-Razzak 